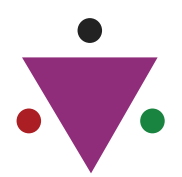

IJCRR

Section: Healthcare Sci. Journal Impact Factor: $6.1(2018)$ ICV: 90.90 (2018)

Scopus

\title{
Efficacy of Tramadol in Comparision with Diclofenac in Ureteric Colic Patients Brought to a Medical College in Central India- A Prospective Observational Study
}

\author{
Shadma Quazi ${ }^{1}$, Varsha Gajbhiye ${ }^{2}$, Sharjeel Khan ${ }^{3}$, Shailesh Nagpure ${ }^{4}$
}

Tutor, Department of Pharmacology, Datta Meghe Medical College, (Datta Meghe Institute of Medical Sciences) Wanadongri, Hingna, Nagpur Maharashtra, India; ${ }^{2}$ Associate Professor, Department of Pharmacology, Datta Meghe Medical College, (Datta Meghe Institute of Medical Sciences) Wanadongri, Hingna, Nagpur Maharashtra, India; ${ }^{3}$ Assistant Professor, Department of FMT, Datta Meghe Medical College, (Datta Meghe Institute of Medical Sciences) Wanadongri, Hingna, Nagpur Maharashtra, India; ${ }^{4}$ Associate Professor, Department of Pharmacology Jawaharlal Nehru Medical College, Datta Meghe institute of Medical Science, Sawangi, Wardha.

\section{ABSTRACT}

\section{Objective:}

1. To estimate the amount of patient exposed to NSAID i.e. Diclofenac and opioid i.e. Tramadol and towhat extent drugs are properly used, overused.

2. Our thesis aimed to provide a comparative overview of NSAID's analgesic efficacy i.e. Diclofenac, i.e. Opioids Tramadol and aimed at evaluating which type of drug is ideally suited in our Emergency Department (ED) for pain relief in acute renal colic.

Methods: The study was planned as a prospective observational study conducted in Ureteric colic Patients receiving analgesics Tramadol and diclofenac in their management. Diagnosis was established by non-contrast abdominal CT. Fifty patients were selected for the study, of which 28 were given Diclofenac and 22 Tramadol. Data was analysed by using Microsoft excel sheet and descriptive statistics.

Results: Characteristics of patients such as average stone size and degree of hydronephrosis at the time of enrolment were close to those of previous investigations. Diclofenac was slightly more effective in minimizing pain intensity at 30 minutes as calculated on a $10 \mathrm{~cm}$ visual analog scale than Tramadol. A reduction in pain frequency of over 50 per cent was reported in 56 per cent of patients diagnosed with Diclofenac and 44 per cent of patients treated with Tramadol $(P<0.05)$. Further Tramadol community patients required analgesia for the release $(p<0.05)$. Taking into fact all the study variables, Diclofenac has an enhanced analgesic efficacy than Tramadol in all aspects.

Conclusions: The drug Diclofenac i.e. NSAID is more effective than Tramadol i.e. OPOID, as a single agent for treating ureteric colic patients. Tramadol can be an option in situations where contraindications prohibit Diclofenac from being used.

Key Words: Ureteric colic, NSAIDs, Opioids, Analgesia, Emergency department

\section{INTRODUCTION}

Renal colic is normal in emergency departments (ED) to pose clinical issue. Acute flank pain characterizes the classic clinical picture, which often is often fluctuating and radiating to the groin and scrotum in males. ${ }^{1}$ Pain is typically caused by upper ureteric obstruction which increases intrapelvic pressure and moreover increases stress in the ureter along with renal pelvic walls. The increasing within the pelvis additionally induces local stimulus for synthesis of prostaglandins i.e E2 Prostaglandin E2 is the most commonly known active diuretic which triggers subsequent dilatation of vessels and induces a diuresis contributing to raisepressure within the pelvis further increasing the pain severity. ${ }^{2}$ Apart from the aforementioned, prostaglandin acts directly on the ureter to cause smooth muscle spasms. The ureteric stone further induces peristalsis of the ureter with an effort to transfer the stone. The oedema and inflammation of

\section{Corresponding Author:}

Dr. Varsha Gajbhiye, Associate Professor, Department of Pharmacology, Datta Meghe Medical College, (Datta Meghe Institute of Medical Sciences), Wanadongri, Hingna, Nagpur Maharashtra, India; Mob: 980959395; Email: ambad.sawan@gmail.com

ISSN: 2231-2196 (Print)

Received: 01.06 .2020
ISSN: 0975-5241 (Online)

Revised: 24.06 .2020
Accepted: 06.07 .2020
Published: 21.07 .2020 
the ureter causesfurther narrowing of ureter where the stone was embedded resulting in further ureteric spasm ${ }^{3}$. Therefore having knowledge of position of ureter and its association to nearby structures are significant in theoretical as well as clinical viewpoint to reserve renal functions, ${ }^{4}$ which to be taken in consideration on basis previous investigations.

Renal colic pain is very painful and can be worse than childbirth or broken bone pain, the reason for the immediate and full pain management is considered to be the most critical point in renal colic therapy approaches ${ }^{5}$. The pain due to renal colic is one of the most severe types of pain requiring timely and effective care. ${ }^{6}$

The key problem in the ED treatment of these patients is sufficient pain management. ${ }^{1,3,4}$. The pain relief drugs are the only treatment modalities needed in about 90 percent of patients. ${ }^{1}$ Rapid and efficient management of pain is an important priority in these patients. But the most therapeutic analgesic treatment yet remains undecided. ${ }^{8}$ The management of pain in patients with ureteric colic depends on the intensity of pain. ${ }^{1}$ Conservative treatment failure depends primarily on two parameters: stone size and urinary tract stone position ${ }^{1}$. The stronger analgesia is required in mild to extreme pain. Nonsteroidal anti-inflammatory drugs (NSAIDs) and opiates are safe options ${ }^{9}$ but in cases of renal failure NSAIDS are not recommended in patients as they may affect the kidney. ${ }^{9}$ Other analgesics can be used in these cases. ${ }^{1}$ Diclofenac is a popular NSAID used in renal colic patients. ${ }^{1}$ The NSAIDs have the potential and effective benefit of decreasing the ureteral smooth muscle tone which ultimately targets the process by which pain is thought to occur. ${ }^{10}$ Tramadol is a type of semi-synthetic opiate which has the untoward benefit of causing lessdepression of respiratory centre $(<1$ percent 0 and has an additional analgesic effect. ${ }^{11,12}$ The various types of opioid analgesics and NSAIDs remain the main stay of acute renal colic care. ${ }^{13}$

The analgesics administration by the oral route in the patient with renal colic is not the most preferred route because these patients may develop vomiting, nausea and because of the slowlydeveloping analgesic effect. Sothe parenteral is the preferred route either give it intravenously (IV) or intramuscularly (IM). The theoretical benefit of the IM route is that it does not require supervision and therefore can be performed easily even in a crowded ED. ${ }^{1}$ There is an inverse proportion between the size of stone and its spontaneous passing. In general, pain is calculated by the "visual analog level" (VAS) of 1 , no pain to 10 . VAS $<4$ usually refers to mild discomfort. (1-8)

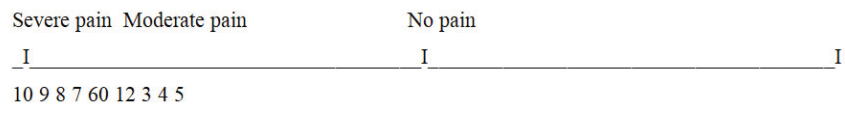

Figure 1: Visual Analogue Pain Score $0-10:{ }^{4}$
Analgesic opioids, e.g. morphine and pethidine, are routinely effective in the acute cases. They have various side effects ranging from nausea, vomiting to respiratory depression and hypotension. ${ }^{14}$ Opioids are strong central analgesic agents that are used to provide a controlled anaesthesia technique..$^{1-3}$ It is possible to achieve the post op pain relief by different approaches, including the use of systemic opioids and regional anaesthesia with intrathecal or epidural opioids, or local anaesthesia. The patient-controlled analgesia (PCA) device is considered to be the best solution for pain analgesia on demand using systemic opioids. ${ }^{15}$ The opioid pain relieverssuch as morphine and pethidine are highly effective for the severe types of pain. ${ }^{10}$ At the same time codeine and dihydrocodeine are of less potency opioids than morphine and better used for mild to moderate type of pain. ${ }^{13}$

Tramadol is an opioid analgesic with less side effects of opioids, especially less respiratory depression, constipation and addictive potential. Intravenous, subcutaneous, intramuscular, and oral preparations are available.For normal postoperative pain, tramadol is as reliable as morphine but it tends to be less effective in extreme acute pain. Popular adverse effects include sedation, dizziness, nausea, dry mouth, and. Tramadol $100 \mathrm{mg}$ has been shown to be as effective as pethidine $50 \mathrm{mg}$ when used for treating renal colic, ${ }^{16}$ it is also said theoccurrence of vomiting and nausea is further more in patientsreceiving tramadol in comparison withbutorphanol. ${ }^{17}$

TRAMADOL is amongst the atypical opioids with core action. Tramadol [tramadol hydrochloride:(1RS, 2RS)-2[(dimethylamine) methyl-1-(3 methoxyphenyl)-cyclohexanol $\mathrm{HCl}$ ] is a synthetic codeine analogue of 4-phenyl-piperidine. ${ }^{19}$

The oral drug is absorbed in the upper small intestine is 95 to 100 percent in relation to human pharmacokinetics and the bioavailability of the drug is 70 percent after single doses (due to an approximately 30 percent first-pass metabolism) and 90 percent in multiple dose studies with a steady state achievement within 36 hours. ${ }^{19,20,21,22}$ Tramadol has a high tissue similarity with the delivery volume of 306 and 203 litres after oral and intravenous (IV) administration, respectively. ${ }^{8}$ Approximately 20 per cent of the drug is bound to protein. ${ }^{23}$ Tramadol is a synthetic opioid analgesic with very fewopioid side effects, particularly it causes lesser respiratory depression, less constipation and has negligible amount of addiction potential. ${ }^{24}$

NSAIDs are the drugs of choice at relieving pain from acute renal colic as much as opioids. While the patients on morphine report more pain relief at 10 minutes but there is no substantial difference by $20-30$ min. $^{25}$

The NSAIDs act by prostaglandin synthesis inhibition. It avoids afferent arteriolar vasodilation and increasing the permeability of vascular membrane which causes diuresis and 
elevates pelvic pressure in the renal system. This also results in decrease in muscular oedema, inflammation and ureteric activity. ${ }^{13}$

Many NSAIDs are available but the key differences between them are their efficacy and form of side effects which are primarily gastric irritation and ulceration. ${ }^{10}$

Naproxen and diclofenac have been associated with lesser side effects with greater analgesia. Indomethacin is well compared with naproxen but has a higher side effects including vomiting, dizziness and gastrointestinal disorders. The oral diclofenac and oral / rectal indomethacin have been shown to be effective in reducing the number of new colic episodes. ${ }^{13}$

Diclofenac, tenoxicam, and lornoxicam are used as intravenous preparations. They have the quickest onset of action, but this is shadowed by a greater risk of side effects ${ }^{13}$, including nausea, vomiting, a chest discomfort, giddiness, weakness, and general malaise. ${ }^{13}$ NSAIDs vary in their selectivity to suppress various forms of cyclooxygenase. ${ }^{14}$

Our analysis aimed at contrasting the efficacy of NSAID, i.e. Diclofenac and opioids i.e. Tramadol and aimed at deciding the fact as the type of medicationthat ismaximum effective aimed at treating pain in acute ureteric colic in Emergency Departments.

\section{MATERIALS AND METHODS}

It is a study which is hospital based and conducted in the Dept. of Pharmacology at Datta Meghe Medical College, Shalinitai Meghe Hospital and Research Centre Nagpur in collaboration with Jawaharlal Nehru Medical College, Datta Meghe Institute of Medical Sciences, Sawangi, Wardha,Maharashtra.The study was planned as a prospective observational study. Duration of study was July 2019 until June 2020 (1 year), fifty clinically diagnosed ureteric colic patients were allocated to the study. The study was permitted by the institutional ethical Committee of the hospital.

Study population: Both the outdoor \& indoor patients who received analgesics Tramadol and diclofenac in any form (i.e. oral, topical or parenteral)

\section{Inclusion criteria:}

1. Both males and females from 18 years of age to less than 65 years of age.

2. Ureteric colic Patients receiving analgesics Tramadol and diclofenac in their management

3. Patient who gave informed consent with their diagnosis as ureteral calculus.

\section{Exclusion criteria:}

1. Patient not willing to give consent.
2. Patients taking medicine for any other systemic diseases. (e.g: APD, CKD, diabetes, hypertension)

3. Pregnancy and lactating mothers.

4. Patients allergic to the study drugs.

5. Patients who had taken analgesics beforesix hoursadmission, were excluded due to the possible effect of concealing the pains.

\section{Sample Size:}

Sample size was calculated using previous studies knowledge and came out to be 50 .

\section{Data collection:}

Source of data- Data was generated from

1. Casualty Software of the centre.

2. Hospital records which include OPD paper/ IPD papers/ Investigation reports.

The following parameters were noted-

1. Patient's demographic data: Name, Age, Sex, Address, Registration No.

2. Date of attending outpatient or inpatient emergency department.

3. Chief complaints and diagnosis.

4. Details of each analgesic prescribed
a. Drug Name
b. Dosage form of drug. (oral,topical,parenteral)
c. Dose
d. Strength of drug preparation (in case of topical)
e. Route of administration
f. Frequency of administration
g. Duration of each analgesic prescribed

Those patients who completed the process of informed consent,received the two drugs were assigned to the two study groups.

As Group1: Patients receiving Diclofenac 75mg.

Group2: Patients receiving Tramadol 100mg.

The patient was asked to score the initial pain severity from 1 to 10 (fig.1) on a Visual Analog Score14 (VAS). It included one with mild to extreme pain (VAS score $>$ or $=4$ ). The patients were randomized to take the study drugs with analgesic doses; either Diclofenac $75 \mathrm{mg}$ (Group 1) or Tramadol $100 \mathrm{mg}$ (Group 2). The pain was assessed at two points before starting the medication and 30 minutes later on. Theassessment was often completed by doctors who were unaware to the consignment of care. Rescue analgesia with intravenous morphine was provided once pain management was not attained $(<50$ per cent decrease in VAS score). Morphine administered was $0.1 \mathrm{mg} / \mathrm{kg}$.

\section{Study procedure}

All patients were examined, and the resident of the emergency room received a comprehensive history, and the results 
were reported in the form of data collection. The period of start and length of the discomfort and the related indications were documented, thru the amount and dates of previous incidents, the removal of calculus and the preceding stone documentation by imaging to confirm nephrolithiasis or hydronephrosis. Vitals were noted.

Study's primary endpoint was the severity of pain assessed upon admission and 30 minutes after the operation. To measure pain severity a Visual Analog Scale (VAS) was used.It consists of a $10 \mathrm{~cm}$ horizontal scale oscillating from no pain to unbearable pain (1 to 10). A second treatment was administratedto those who had no acceptable pain respite within 30 minutes. The adverse effects of both the drugs was recorded and comparativeanalysis done between the groups. Persons those measuring the consequencestayed blinded to study groups.

\section{Statistical analysis}

Microsoft excel sheet and descriptive statistics were used to evaluate the data The difference will be calculated; ' $t$ ' test will be used for control variables. ANCOVA and logistic regression can also be used to analyse the impact of few variables on VAS. ${ }^{26}$

A two sided p-value $<0.05$ was considered as statistically significant.

\section{RESULTS}

Flow chart:

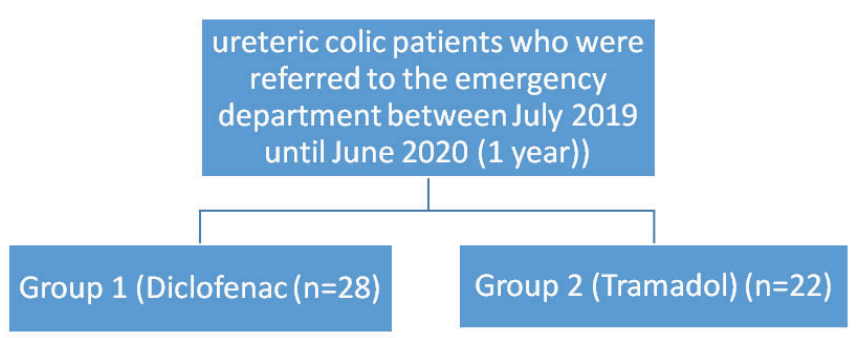

Figure 2: Consort flow diagram of the study.
Table 1: Showing baseline features of the study patients

\begin{tabular}{lcccc} 
Groups & Age Array & Mean Age & Male & Female \\
1 & 18 to 65 & 41.5 & 18 & 10 \\
2 & 16 to 64 & 41 & 14 & 8 \\
\hline
\end{tabular}

The 50 patients surveyed considered 32 males and 18 females having an average mean age as 41.25 ages (range, 18 - 65 ages) (Table 1). Baseline features consisting of age, sex and pain severity, indicate no substantial variance between the two groups at presentation time. The number of males being more than female with male: female ratio of 16:9. Baseline creatinine level was $92 \pm 17$ (normal range 60-110mmol/1) for both groups.

Table 2: Showing Mean VAS score at diverse timeinterims of the two groups

\begin{tabular}{lccccc} 
& \multicolumn{5}{c}{ Time interim } \\
Group & o min & 10 min & $30 \mathrm{~min}$ & $60 \mathrm{~min}$ & 2 hours \\
1-Diclofenac & 8.55 & 8.20 & 4.05 & 2.55 & 2.00 \\
2-Tramadol & 8.65 & 5.00 & 3.40 & 4.00 & 4.50 \\
P value & $<0.05$ & $<0.05$ & $<0.05$ & $<0.05$ & $<0.05$ \\
\hline
\end{tabular}

Table 2 Summarizing pain level results between the two classes. The mean value of the VAS scale was 8.55, 8.65, for Diclofenac and Tramadol respectively, and at the time of presentation (zero minute) they were equivalent for the whole group $(\mathrm{p}>0.05)$. Important decrease in mean VAS score after 10 minutes of administration was shown: 5.00 for group two. One VAS score (4.05) was observed at 30 i.e. decrease in group one. Laterafter 60 minutes of drug administration, similar another decrease in the VAS score was observed, 2.55, 4.00, respectively for groups one and tworespectively. After 60 minutes and 2 hours respectively, group receivingtramadol showed a starting increase in VAS score as 4.00 and 4.50 which could require another dose or medication in certain patients.

Table 3: Showing adverse effects of two drugs

\begin{tabular}{lcccccc} 
Group & \multicolumn{7}{c}{ Adverse effects (number of Patients) } & urine retention \\
& nausea & vomiting & dizziness & dry mouth & rash & ury \\
1-Diclofenac & 1 & 2 & 1 & 0 & 0 & 0 \\
2-Tramadol & 10 & 8 & 4 & 0 & 0 & 1 \\
\hline
\end{tabular}


Table 3 Showing side effects with both classes of medications. The main adverse effects of Tramadol include vomiting, nausea and giddiness while, mild adverse effects were dry mouth and skin rash.

Of the 50 patients under review, 48 completed the review (the other 2 patients who obtained Diclofenac from group1 and Tramadol from the group 2 could not be involved in the research due to deficiency of reliable pain assessment.Total Twenty-seven patients received Diclofenac (group 1) and Tramadol (group 2) received twenty-one subjects. All results during the patient's stay in the ED were measured. There were no major side-effects reported in either group. (table3)

\section{Table 4: Showing Average VAS before and after Treat- ment with the Study Drugs}

\begin{tabular}{lccc} 
& VAS before & VAS after & $\begin{array}{c}\text { The Delta Pain } \\
\text { Reduction (\%) }\end{array}$ \\
Diclofenac & $8.8 \pm 1.5$ & $4.2 \pm 2.6$ & $52 \%$ \\
Tramadol & $8.6 \pm 1.3$ & $5.6 \pm 2.9$ & $35 \%$ \\
\hline
\end{tabular}

The averaged VAS score at presentation was $8.8 \pm 1.5$ for group 1 and $8.6 \pm 1.3$ for group 2 .

\section{Pain reduction}

The pain was relieved in 64 per cent of Diclofenac-treated patients and in 49 per cent of Tramadol-treated patients $(\mathrm{P}<0.05)$ (Fig. 1). In patients diagnosed with Diclofenac, the reduction in delta pain was greater than in patients treated with Tramadol in an average of 17 per cent (Table 4).So; it was appropriate to treat more patients in the Tramadol community with rescue analgesia $(\mathrm{P}<0.05)$, as shown in Fig. (2). These results are of significant value. ${ }^{1}$ Fig. (2). The percentage of patients who needed salvage analgesia. ${ }^{1}$

\section{Table 5: Showing the quantitative VAS Score Reduc-} tion

\begin{tabular}{lccc}
\multicolumn{3}{c}{$\begin{array}{c}\text { VAS reduction }>\mathbf{2} \\
\mathbf{N}^{\circ}(\%)\end{array}$} & $\begin{array}{c}\text { VAS reduction }<2 \\
\mathbf{N}^{\circ}(\%)\end{array}$ \\
Diclofenac & $24(87.5 \%)$ & $3(12.5 \%)$ & $27(100 \%)$ \\
Tramadol & $13(61.2 \%)$ & $8(38.8 \%)$ & $21(100 \%)$ \\
Total & $37(74.2 \%)$ & $11(25.8 \%)$ & $48(100 \%)$ \\
\hline
\end{tabular}

The response rate in the Diclofenac group was 87.5 percent, and the Tramadol group was just 61.2 percent. Such findings support our initial assumptions (Table 5).

\section{DISCUSSION}

The patients presenting with acute ureteric colic, the NSAIDs along with OPOIDS being used conventionally for pain relief. ${ }^{1}$
The average age incidence in this study is (32.35 to 40.20 years) with males are more common than females (32 males and 18 females) (Table 1), this finding is proper if we know that renal calculus disease in men is more common 2-3 times in males than females and more common in adults than elderly people and is extremely uncommon in children. ${ }^{27}$

Diclofenac sodium and Tramadol are similarly successful in reducing the severity of pain at 30 minutes patients received substantial pain relief. The difference between the Tramadol category is that the pain level begins explicitly at just 10 minutes [Figure 1]. Cordell et al. find in literature that the NSAIDs group of drugs and opioids group have comparable effectiveness but without any substantial difference in pain strength decrease 20-30 minutes after the administration. ${ }^{28}$

Also with the flexibility of the Tramadol group, the pain escalates at 60 minutes and two hours'intermissions due to a sudden decrease in the pain severity being found only after 10 minutes, which could involve a further dose. Considering the fact of needs for extra doses, Holgate et al. have a significant findings that opioid group both pethidine and morphine, having 6-10 times more potency than tramadol, have better levels of additional analgesia than NSAIDs group. ${ }^{29}$ Diclofenac dry mouth, nausea, diarrhoea, and rash are fewer of the side effects of medications, whereas diarrhoea and dizziness are frequent side effects of tramadol. These drugs have the side effect of making NSAIDs the first alternative. ${ }^{30}$

Prospective randomized controlled trials have shown that drugs belonging to NSAIDs group are at least as effective as Opiates. ${ }^{31}$ Tramadol has been shown to be an effective, wellaccepted treatment for reducing renal and ureteric colic pain. Tramadol tends to produce very less amount of constipation as well as respiratory depression rather than the strong opioid analgesic doses. ${ }^{32}$

In this report, and after evaluating most reports of renal colic pain relief, we first pick Diclofenac as the most operational NSAIDs in relieving ureteric colicky pain due to its high analgesic effects and with less side effects than morphine. ${ }^{33}$ Secondly, in most OPD facilities in hospitals, fast-acting opioid-like drugs are available with less side effects than other opioids such as morphine and pethidine but they have the side effects to cause vomiting, respiratory distress and drug dependency and addiction ${ }^{34}$ also known as Hypersensitivity reactions(type 1). ${ }^{35}$ Thirdly, in several trials, the combination therapy found to be a better option than monotherapy in obtaining a rapid and sustained pain relief, besides minimizing the need for rescue analgesia in general practice. ${ }^{5}$ As for each of the above, few published trials investigate the potential synergistic impact of NSAIDs and opioids.

To our knowledge, there were not many previous studies comparing drug NSAIDs and tramadol given in the setting as an analgesic treatment in ED in renal colic. 
According to our findings, intramuscular Diclofenac tends to be more successful than intramuscular Tramadol as a single agent for treating renal colic. Diclofenac's beneficial effect was not affected by the occurrence or degree of severity of hydronephrosis, nor by the position or scale of the calculus. All of these factors were identical for both treatment groups.

The distinction between our research and others is how we provided pain relief evaluations. In our research, we determined response as pain relief from the baseline before drug administration of at least 50 percent. The other studies have used a quantitative reduction of the VAS score. ${ }^{36,37}$ This has used more than 2 baseline VAS score reductions to classify successful response. Pursuant to this approach we evaluated our findings retrospectively. The updated results showed: the response rate in the Diclofenac group was 87.5 percent, and the Tramadol group was just 61.2 percent. Such findings support our initial assumptions (Table 5).

Further, due to the aged population and increasing healthcare expenditures, fitness promotion and infirmity anticipation is emphasised, to offer primary intercession or multidisciplinary managing at the primary health level, so in future it is important to categorise at risk patients people based on ill-health ${ }^{38}$ so that proper drug and dose can be administered.

\section{Limitations:}

The study conveys some limitations as:

1) The number of research cases was smaller in number which bounds the statistical validity of some findings. A bigger study would be needed to study important subgroups and various complications

2) If the two drugs were given by same route, hypothetical variability would have affected the results.

3) General analysis of means rather than medians isdoubtful to introduce bias unless the delivery of scores is rigorously skewed.

4) As a practice in emergency departments is to titrate opioids to effect rather than to give single dose, thus limiting the applicability of our results to day to day practices.

Thus, a wide diversity of drug types and dosages used in the studies mark it problematic to identify suitable medicating schedules for medical practice.

\section{CONCLUSIONS}

Our study of Diclofenac has now proved to be superior to Tramadol for pain relief in patients with ureteric colic. Tramadol may be used as an alternative when contraindications prevent the use of Diclofenac, but as stated in this report, less than half of the subjects treated with Tramadol have achieved good pain control.
Single doses of NSAIDs and opioids provide pain relief for patients suffering from acute ureteric colic. However, patients receiving NSAIDs report a greater reduction in pain levels, and are less likely to require more short-term analgesia. Opioids, particularly Tramadol, are associated with greater rates of side effects most common being vomiting than NSAIDs therefore,we prescribe an NSAID instead of an opioid.

With less side effects of the opioid form and less dependence risk, Tramadol can be a safe medication in the treatment of ureteric colic patients, particularly in circumstances where NSAIDs are contraindicated, or who have a history of drug abuse in patients.

Acknowledgement: Authors acknowledge immense help received from Datta Meghe Medical College, (Datta Meghe Institute of Medical Sciences) for conducting this study

\section{Source of Funding and sponsorship: Nil.}

Conflicts of interest: There are no conflicts of interest.

\section{REFERENCES}

1. Shaden Salameh, Nurit Hiller, Meir Antopolsky, Fedaa Ghanem et al. Diclofenac versus Tramadol in the Treatment of Renal Colic: A Prospective, Randomized Trial. The Open Emergency Medicine Journal, 2011, 4, 9-13.

2. Anna Holdgate, Tamara Pollock. Systematic review of the relative efficacy of non-steroidal anti-inflammatory drugs and opioids in the treatment of acute renal colic. BMJ. 2004 Jun 12; 328(7453): 1401.

3. Crowley, A.R.; Byrne, J.C.; Darracott Vaughan, E.; Marion, D.N. The effect of acute obstruction on ureteral function. J. Urol. 1990, 143:596-599.

4. Roy, M., B.R. Singh, U.L. Gajbe, and P. Thute. "Anatomical Variations of Ureter in Central India: A Cadaveric Study.” Journal of Datta Meghe Institute of Medical Sciences University 12, no. 4 (2017): 277-79.

5. Hussein Oudah, Al-Jasmawy, Adel Ibraheem Muhi Ali, Abdulhaleem Kadhim et al. Efficacy of Combined Tramadol with Diclofenac in Comparison with Monotherapy Treatment Using Buscopan Diclofenac or Tramadol in Renal Pain Control, Medical Journal of Babylon Vol. 12- No. 4:1070 -1076 , 2015.

6. Shokeir AA. Renal colic: new concepts related to pathophysiology, diagnosis and treatment. Curr Opin Urol. 2002; 12(4):2639.

7. Teichman JM. Clinical practice. Acute renal colic from ureteral calculus. N Engl J Med. 2004; 350(7):684-93.

8. Holdgate A, Pollock T. Systematic review of the relative efficacy of non-steroidal anti-inflammatory drugs and opioids in the treatment of acute renal colic. BMJ 2004; 328(7453): 1401.

9. Dieppe P, Bartlett C, Davey P, et al.. Balancing benefits and harms: the example of non-steroidal anti-inflammatory drugs. BMJ 2004; 329(3): 31-4.

10. Cole RS, Fry CH, Shuttleworth KE. The action of the prostaglandins on isolated human ureteric smooth muscle. Br J Urol 1988; 61(1): 19-26

11. Grond S, Sablotzki A. Clinical Pharmacology of Tramadol. Clin Pharmacokinet 2004; 43(13): 879-923. 
12. Mortelmans LJM, Desruelles D, Baert JA, et al.. Use of tramadol drip in controlling renal colic pain. J Endourol 2006; 20(12): 1010-5.

13. Kim Davenport, Anthony G. Timoney and Frank X. Keeley. Conventional and alternative methods for providing analgesia in renal colic 2005 BJU International, 95, 297-300.

14. Porpiglia F, Destefanis P, Fiori C, Fontana D. Effectiveness of nifedipine and deflazacort in the management of distal ureter stones. Urology. 2000; 56(4):579-82.

15. Pallavi Ahluwalia, Fauzia Rehman, Amit Ahluwalia. Comparison of Butorphanol Tartrate and Tramadol Hydrochloride for Post-Operative Pain Relief Following Abdominal Surgery: A Prospective, Randomized, Double-Blind Study. International Journal of Scientific Study | December 2015 | Vol 3 | Issue 9.120124.

16. Salehi M, Ghaserni H, Shiery H, Roshani A, Khosropanah I, Asgari A. Intramuscular tramadol versus intramuscular pethidine for treatment of acute renal colic. J Endourol 2003; 17 (Suppl. 1): A243.

17. Palan, A., and N.K. Agrawal. "Control of Intraoperative Shivering under Spinal Anaesthesia- A Prospective Randomized Comparative Study of Butorphanol with Tramadol.” Journal of Krishna Institute of Medical Sciences University 6, no. 1 (2017): 57-65.

18. Dayer P, Desmeules J, Collart L. Pharmacology of tramadol. Drugs 1997; 53 (Suppl 2):18-24.

19. Bamigbade TA, Langford RM. The clinical use of tramadol hydrochloride. Pain Reviews 1998; 5:155-182.

20. Duthie DJR. Remifentanil and tramadol. Br J Anaesth 1998; 81:51-57.

21. Stamer UM, Maier C, Grond S, Veh-Schmidt B, Klaschik E, Lehmann KA. Tramadol in the management of post-operative pain: a double-blind, placebo- and active drug-controlled study. Eur J Anaesthesiol 1997; 4:646-654.

22. Lehmann KA. Tramadol in the management of acute pain. Drugs 1994; 47 (Suppl 1):19-32.

23. Bono AV, Cuffari S. Effectiveness and tolerance of tramadol in cancer pain: a comparative study with respect to buprenorphine. Drugs 1997; 53 (Suppl 2):40-49.

24. Mehdi Shirazi, Mehdi Salehipour, Mohammad Amin Afrasiabi, Alireza Aminsharifi. Analgesic Effects and Safety of Desmopressin, Tramadol and Indomethacin in Patients with Acute Renal Colic; A Randomized Clinical Trial. Bull Emerg Trauma 2015;3(2):41-45.

25. Cordell WH, Larson TA, Lingeman JE et al.. Indomethacin suppositories versus intravenous titrated morphine for the treatment of ureteral colic. Ann Emerg Med 1994; 23: 262-9.
26. E. A. SHIPTON. Tramadol-Present and Future, Anaesth Intensive Care 2000; 28: 363-374.

27. Portis AJ CP. Diagnosis and initial management of kidney stones, Sundaram. Am Fam Physician. 2001; 63(7):1329-38.

28. Cordell, W.H.; Larson, T.A.; Lingeman, J.E.; Nelson, D.R.; Woods, J.R.: Burns, L.B.; Klee, L.W. Indomethacin suppositories versus intravenous titrated morphine for the treatment of ureteral colic. Ann. Emerg. Med. 1994, 23, 262-269.

29. Holdgate A, Pollock T. Nonsteroidal anti-inflammatory drugs (NSAIDs) versus opioids for acute renal colic. Cochrane Database of Systematic Reviews 2004, Issue 1. [DOI: 10.1002/14651858.CD004137.pub2]

30. Labrecque M, Dostaler LP, Rousselle R, et al.. Efficacy of nonsteroidal antiinflammatory drugs in the treatment of acute renal colic. A meta-analysis [see comments]. Arch intern Med 1994; 154:1381-7.

31. Cordell WH, Wright SW, Wolfson AB, et al.. Comparison of intravenous ketorolac, meperidine, and both (balanced analgesia) for renal colic. Ann Emerg Med 1996; 28(2): 151-8.

32. Grond S, Sablotzki A. Clinical Pharmacology of Tramadol. Clin Pharmacokinet 2004; 43(13): 879-923.

33. Cole RS, Fry CH, Shuttleworth KE. The action of the prostaglandins on isolated human ureteric smooth muscle. Br J Urol 1988; 61:19.

34. Shadma H. Quazi, Sushil K. Varma, Sharjeel H. Khan and et al.. Hypersensitivity reactions to intravenous ferric carboxymaltose in a patient with iron deficiency anemia: A rare case report. International Journal of Basic \& Clinical Pharmacology, May 2018, 7 (5):1036-1039.

35. Davenport K, Timoney AG, Keeley FX. Conventional and alternative methods for providing analgesia in renal colic. BJU Int.2005; 95(3):297-300.

36. Olsen JC, McGrath NA, Schwarz DG, et al.. A double-blind randomized clinical trial evaluating the analgesic efficacy of ketorolac versus butorphanol for patients with suspected biliary colic in the emergency department. Acad Emerg Med 2008; 15(8): 718-22.

37. Priya, N., Y.R. Lamture, and L. Luthra. "A Comparative Study of Scalpel versus Surgical Diathermy Skin Incisions in Clean and Clean-Contaminated Effective Abdominal Surgeries in AVBRH, Wardha, Maharashtra, India." Journal of Datta Meghe Institute of Medical Sciences University 12, no. 1 (2017): 21-25.

38. Kumar, S., S. Jain, A. Wanjari, and S. Mandal. "Development and Validation of a Modified Frailty Risk Index as a Predictor of Mortality in Rural Elderly People." Asian Journal of Gerontology and Geriatrics 14, no. 1 (2019): 15-22. 